\title{
Genomic epidemiology of multidrug-resistant Gram-negative organisms
}

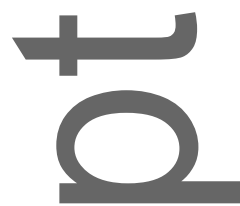

Shawn EHawken ${ }^{1}$ and Evan S Snitkin ${ }^{1,2}$

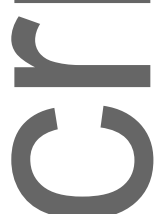

${ }^{1}$ Department of Microbiology and Immunology, University of Michigan Medical School, Ann Arbor, Michigan. ${ }^{2}$ Department of Medicine, Division of Infectious Diseases, University of Michigan Medical School, Ann Arbor, Michigan

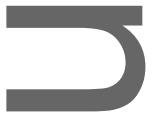

Address for correspondence: Evan Snitkin, PhD, 1520D MSRB I, 1150 W. Medical Center Dr., Ann Arbor, MI, 48109-5680. esnitkin@med.umich.edu

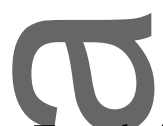

Short title: Translational genomics of resistant Gram-negatives

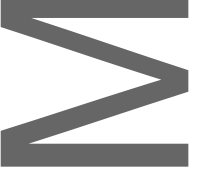

\section{Abstract}

The emergence and spread of antibiotic-resistant Gram-negative bacteria (rGNB) across global healthcare networks presents a significant threat to public health. As the number of effective antibiotics available to treat these resistant organisms dwindles, it is essential that we devise more effective strategies for controlling their proliferation. Recently, whole-genome sequencing has

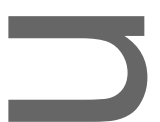

This is the al 1 manuscript accepted for publication and has undergone full peer review but has not been thi wo tl c copyediting, typesetting, pagination and proofreading process, which may lead to differences betm this version and the Version of Record. Please cite this article as doi: $10.1111 /$ nyas.13672.

This article is protected by copyright. All rights reserved. 
emerged as a disruptive technology that has transformed our understanding of the evolution and epidemiology of diverse rGNB species, and it has the potential to guide strategies for controlling the evolution and spread of resistance. Here, we review specific areas in which genomics has already made a significant impact, including outbreak investigations, regional epidemiology, clinical diagnostics, resistance evolution, and the study of epidemic lineages. While highlighting early successes, we also point to the next steps needed to translate this technology into strategies to improve public health and clinical medicine.

Keywords: genomic epidemiology; multidrug-resistant organisms; hospital-associated infection; whole-genome sequencing; antibiotic resistance
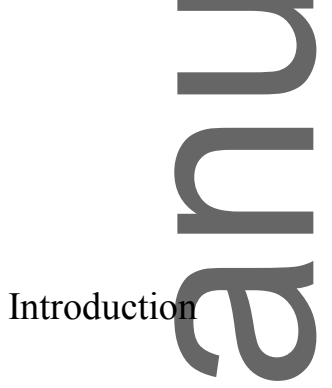

In recent years, multidrug-resistant organisms (MDROs) that are refractory to nearly all available treatments have emerged and spread globally. ${ }^{1,2}$ The inability of drug discovery pipelines to keep up with the pace at which resistance has neutralized existing antibiotics has created an imminent global public health crisis. ${ }^{3,4}$ The threat of MDROs is particularly dire within our healthcare systems, where more than 1 in 25 hospitalized patients have a healthcare-associated infection (HAI) on any given day. ${ }^{5}$ Hospitalized patients have co-morbidities that make them more susceptible to contracting infections and less equipped to combat these infections without the aid of antibiotics. Thus, increases in antibiotic resistance among healthcare-associated pathogens has directly led to increases in morbidity and mortality among affected patients. ${ }^{6-8}$ Recently, the evolution of antibiotic resistance has reached a crucial tipping point with the emergence of pan-resistant organisms that have caused infections untreatable with any available antibiotic. ${ }^{2,9-11}$ In the absence of novel treatments to combat these resistant infections, there is an urgent need for the development of more effective strategies to

This article is protected by copyright. All rights reserved. 
control the spread of MDROs and prevent patients from acquiring infections that are increasingly difficult to treat.

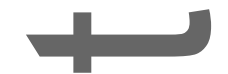

Over the past several decades, healthcare epidemiologists have made significant strides in
tracking the spread of infections within and between healthcare facilities by supplementing traditional gumshoe epidemiology with a diverse suite of molecular typing tools. ${ }^{12}$ Molecular typing methods probe the structure (e.g., pulsed-field gel electrophoresis (PFGE)) or sequence (e.g., multilocus sequence typing (MLST)) of microbial genetic material in order to quantify the relationships among infectious isolates and gauge whether they are plausibly linked by transmission. ${ }^{12-14}$ While much has been learned about the local and global epidemiology of MDROs using molecular typing approaches, all classical techniques are associated with major limitations. First, methods based on genome structure present difficulties in interpretation, because these molecular types (e.g., pulsotype) do not evolve at a consistent rate. ${ }^{14}$ The lack of a quantifiable relationship between variation in molecular type and historical relatedness forces investigators to apply arbitrary cutoffs in evaluating whether two isolates could be epidemiologically linked. ${ }^{13}$ A second issue with classical methods is that there is no single method that performs well at all time scales. For instance, MLST has been shown to be extremely powerful in characterizing regional or global pathogen populations but lacks the resolution to discern transmission patterns within a healthcare institution. ${ }^{15}$ Conversely, PFGE provides resolution sufficient to discern between closely related strains but is often too dynamic to compare pathogen populations in different regions. ${ }^{15}$ Lastly, an important limitation of all classical molecular typing approaches is that they provide no insight into how genetic changes relate to phenotypic differences among strains.

Recently, whole-genome sequencing (WGS) has entered the forefront of molecular epidemiology, providing a one-size-fits-all tool that overcomes virtually all of the limitations of prior methods. First, WGS has been shown to provide sufficient resolution to elucidate transmission pathways within a single institution, while at the same time yielding data that facilitates the placement

This article is protected by copyright. All rights reserved. 
of global pathogen populations in the context of one another. ${ }^{16-18}$ Second, by probing every base pair in the genome, WGS allows investigators to translate genetic differences into historical relationships among isolates by exploiting the molecular clock at which mutations accumulate over time. ${ }^{19,20}$ Having a molecular type that can be related to a molecular clock has allowed investigators to explicitly test whether two strains are linked on epidemiologically relevant time scales while avoiding the need to set arbitrary cutoffs. ${ }^{21}$ Lastly, by interrogating variation across the entire genome, investigators can leyerage phenotypic information from decades of biochemical and genetic experiments to generate hypotheses regarding the phenotypic impact of observed genomic variation. ${ }^{22}$

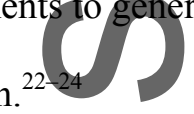

Early work applying WGS to study MDROs has demonstrated the disruptive nature of this technology and led to fundamental insights into the evolution and epidemiology of the most significant healthcare-associated pathogens. Here, we highlight recent applications of WGS to characterize the emergence and spread of Gram-negative MDROs across global healthcare systems. While WGS has had an equally significant impact on Gram-positive MDROs, we focus on Gramnegatives to highlight some of the unique features of this increasingly burdensome class of healthcare pathogens. ${ }^{25,26}$ For each application of WGS, we also explore challenges and opportunities in maximizing the translational impact of this transformative technology in the realms of clinical practice and public health.

\section{Hospital epidemiology and outbreak investigation}

Among the earliest applications of WGS to study the epidemiology of MDROs was to elucidate transmission networks during hospital outbreaks. ${ }^{16,17,27,28}$ Outbreak investigations are initiated when there is a-spike in infections with an MDRO species. A typical investigation consists of case finding, where investigators look for additional patients who might be involved in an outbreak, as well as

This article is protected by copyright. All rights reserved. 
contact tracing, where investigators look for common exposures or contact between patients, with the goal of identifying contaminated infrastructure or pathways of patient-to-patient transmission. Traditional epidemiological investigations are often supplemented with molecular typing in order to narrow the focus to groups of patients that are thought to be part of a transmission chain, based upon their harboring of a related MDRO strain. However, this combination of contact tracing and lowresolution molecular typing has been complicated by the emergence of epidemic MDRO lineages that have become endemic in regional healthcare institutions. In particular, the endemicity of these epidemic lineages is such that it is not uncommon for multiple patients to enter a healthcare institution already colonized or infected with a common strain. Thus, grouping together all patients harboring a common strain will result in patients being grouped together who are not necessarily connected by transmission within a healthcare institution. The inability to accurately group patients linked by transmission can make it difficult to identify contaminated infrastructure or other potentially modifiable factors that are mediating transmission.

Several studies have reported the successful application of WGS to partition patients into transmission elusters when other molecular typing approaches failed. Our first application of WGS to study a hospital outbreak was for an outbreak of MDR Acinetobacter baumannii. ${ }^{28}$ Although our hospital had not had a previous outbreak with MDR A. baumannii, PFGE typing of outbreak isolates indicated that we had three different strain types simultaneously circulating in the hospital. We therefore wondered if this outbreak was due to three contemporaneous introductions into the hospital or if the circulating strain of A. baumannii had evolved in such a way that its PFGE type changed during the course of the outbreak. Application of WGS to representatives of the three outbreak strains led us to the conclusion that two importation events had seeded this outbreak. Two outbreak strains were traced back to an importation event by a single patient, with the variation in strain type believed to be due to large recombination events across the genome. The third outbreak strain was traced back to a non-MDRO strain that had been circulating in the hospital months earlier, and in the intervening

This article is protected by copyright. All rights reserved. 
time had picked up several drug-resistance determinants. In a separate study, Willems and colleagues were able to partition an A. baumannii outbreak into two clusters and show that transmission was largely confined within specific hospital wards, thereby focusing infection control interventions. ${ }^{29}$ Kanamori and colleagues were similarly able to partition an A. baumannii outbreak into clusters due to independent importation events and, similar to our investigation, found that filtering out recombinant regions of the genome was critical to make accurate epidemiological inferences. ${ }^{30}$ The The suceess of WGS in dissecting healthcare outbreaks is not limited to A. baumannii. Stoesser and colleagues applied WGS to isolates from an outbreak of MDR Enterobacter cloacae that primarily affected neonates. ${ }^{31}$ This analysis revealed two separate clusters that were again largely confined to individual units. In addition, one of the clusters matched an isolate retrieved from a soap dispenser, implicating this contaminant as the point source seeding this cluster. Several groups have also applied WGS to study outbreaks of carbapenem-resistant Klebsiella pneumoniae and other MDR Gram-negatives, many of which observed multiple strain importations, followed by the preferential transmission of particular strain types. ${ }^{30-33}$ Upon partitioning multistrain outbreaks into clusters, these studies found that most transmission events could be accounted for by spatiotemporal overlap between patients in the facility, again emphasizing the importance of defining transmission clusters to facilitate insights into transmission pathways. Importantly, most of the aforementioned studies found that the incorporation of WGS data into the outbreak investigation facilitated insights into the origins of circulating strains and pathways of nosocomial transmission that would have been inaccessible with lower-resolution typing methods (e.g., MLST, PFGE).

In addition to grouping patients into transmission clusters, several studies have been able to use WGS to elucidate extremely nuanced insights into the propagation of outbreaks with different MDROs. As mentioned above, multiple groups have reported that time and space overlap on hospital wards can explain the majority of transmissions for organisms such as K. pneumoniae and E. cloacae, which are thought to primarily spread patient-to-patient via healthcare worker contamination. ${ }^{34,35}$

This article is protected by copyright. All rights reserved. 
However, for more environmentally hearty organisms like A. baumannii and Pseudomonas aeruginosa, WGS has allowed for causal links to be made between environmental contamination and ongoing transmission. In studying a prolonged outbreak of A. baumannii, Halachev and colleagues were able to use WGS to link contamination in an operating theater to transmission between patients who otherwise had no overlap in the hospital. ${ }^{33}$ Several groups have linked Pseudomonas isolates from sink drains to isolates taken from patients. ${ }^{36,37}$ While directionality was not clear in many of these cases, one report found that genetically identical isolates persisted in a sink trap months after the linked patient had been in the room, demonstrating at the very least that infection-causing isolates can persist in the hospital environment for extended periods of time. ${ }^{36}$

Lastly, WGS has also yielded nontrivial insights into the structure of transmission networks. Applying WGS to an outbreak of carbapenem-resistant K. pneumoniae allowed us to demonstrate the role of asymptomatic carriers in outbreak propagation. ${ }^{17}$ In particular, we observed that, despite a 3week gap in infections following discharge of the index patient, there had in fact been multiple transmissions from this index patient that seeded an outbreak that affected 18 patients. This observation led to the implementation of more rigorous surveillance culturing, which was critical in identifying and isolating all asymptomatically colonized patients and stopping the outbreak. In applying WGS to an outbreak of $P$. aeruginosa, Willmann and colleagues found evidence for the disproportionaterole of a few super-spreaders in propagating the outbreak strain. ${ }^{36}$ Future insights such as these into the structure of transmission networks for different MDROs will be critical in identifying and properly managing high-risk patients.

While these early studies show how powerful WGS is for outbreak investigations, there are still important challenges that need to be considered. First, several groups, including ours, have reported how intra-patient genetic heterogeneity of colonizing and contaminating populations can confound accurate descriptions of transmission networks. ${ }^{17,21}$ The impact of this is still not fully appreciated, but the potential for many MDRO species to colonize hosts for months or years raises the

This article is protected by copyright. All rights reserved. 
possibility that certain patients may harbor extremely diverse colonizing populations. ${ }^{38-42}$ Moreover, the potential for multiple acquisitions in high-transmission settings has been documented and can also confound transmission inference. ${ }^{43-45}$ While there are both analytical and sequencing-based strategies to deal with these issues, they result in decreased power and increased cost, respectively. ${ }^{46-48}$ One solution to combat decreased power of genetic inferences is to supplement transmission- inference pipelines with comprehensive location or contact-tracing data. ${ }^{49}$ A second solution is to apply methods that account for potential intra-host diversity and uncertainty surrounding the potential transmission, events when constructing transmission networks. ${ }^{46,47}$

Another challenge in standardizing WGS for clinical applications is agreement in the field regarding best practices and common analytical frameworks. Likely, the optimal framework will depend on the question at hand, where computationally friendly pipelines like WG-MLST might be preferable for real-time analyses, while more sophisticated phylogenetic approaches that take full advantage of genomic data can be applied in retrospective analyses. ${ }^{46,47,50,51}$ A related issue is coming to a consensus as to whether the establishment of concrete variant thresholds is appropriate for evaluating whether two patients are plausibly linked by transmission. ${ }^{16,19,49,52}$ Owing to the aforementioned issue of increased variation due to prolonged asymptomatic colonization of patients, it is unlikely that a hard variant cutoff that is not overly conservative will work in all situations. ${ }^{53,54} \mathrm{We}$ believe that a more viable solution for distinguishing between transmission and importation is enacting more comprehensive sequencing of regional isolate collections, such that isolates from within a facility can be placed into a broader regional context. ${ }^{55,56}$ Finally, one must consider whether there is benefit to having WGS embedded in clinical microbiology labs for real-time investigation or whether retrospective investigations by healthcare researchers and public health laboratories are sufficient. ${ }^{57}$ To answer this question will require well-conceived and designed studies that quantify the benefits of real-time sequencing. ${ }^{58}$

This article is protected by copyright. All rights reserved. 


\section{Regional epidemiology at different geographic scales}

While the application of WGS to understand intrafacility transmission has the potential to reduce infection rates by stemming nosocomial transmission, it is increasingly appreciated that the connectivity of healthcare networks will ultimately necessitate a regional approach to infection control. ${ }^{59}$ Such a regional approach will require understanding the structure and dynamics of pathogen populations at different temporal and geographic scales. As the cost of sequencing has decreased and allowed for the application of WGS to large strain collections, it has become clear that a genomics approach can yield unparalleled insights into pathogen populations at local, regional, and global scales.

In addition to targeted sequencing of suspected outbreaks, WGS has been applied more broadly at single institutions to discern local pathogen population structure and gauge the relative impacts of importation and transmission within healthcare facilities. To try and understand an observed increase in carbapenem-resistant Enterobacteriaceae (CRE) at their institution, Pecora and colleagues sequenced all CRE infection isolates over a 3-year period. ${ }^{60}$ Genomic comparison revealed that there was little transmission and that incidence of CRE at this institution was primarily driven by the sporadic importation of organisms harboring diverse mobile resistance elements. Mellmann and colleagues took this to the next level by sequencing all methicillin-resistant Staphylococcus aureus (MRSA), vancomycin-resistant enterococci (VRE), and resistant Gram-negative bacilli (rGNB) at their institution to discern the relative impact of transmission and importation. ${ }^{58}$ Their genomic investigation revealed that there was little transmission of $\mathrm{rGNB}$, which led to modification of infection-eontrol procedures to more effectively allocate resources. Importantly, the authors then applied WGS to validate that their procedural modifications did not have negative consequences on transmission rates. In the ultimate display of sequencing power, Roach and colleagues indiscriminately sequenced every clinical isolate taken from ICU patients over the course of a year. ${ }^{43}$ Analysis of 1229 genomes from 391 patients revealed an unexpected level of species and strain

This article is protected by copyright. All rights reserved. 
diversity in the hospital and painted a picture of overall low transmission rates, with a handful of successful lineages being observed in multiple patients.

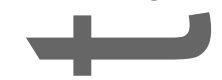

While sequencing isolate collections from single institutions provides insights into what is happening within the confines of a facility, understanding the ultimate origin of MDROs circulating within hospitals will require sequencing and analysis of regional isolate collections. Moradigaravand and colleagues recently took such a regional approach to understand the population structure of three MDR members of the Enterobacteriaceae family: Enterobacter cloacae, Serratia marcescens, and Klebsiella pneumoniae. ${ }^{56,61,62}$ In contrast to MDR K. pneumoniae, in which epidemic clones dominate specific regions, E. cloacae and $S$. marcescens both exhibited a polyclonal structure across 37 hospitals in the United Kingdom and Ireland, indicating the convergent emergence and spread of multiple MDR lineages. This finding contrasts to a recent report by Hargreaves and colleagues, who observed the emergence and spread of a lineage of bla-KPC-carrying E. cloacae across multiple hospitals in North Dakota and Minnesota. ${ }^{63}$ Thus, polyclonal population structures are not necessarily a static feature of a given MDRO species, with the acquisition of a key resistance determinant potentially leading to the emergence of clonal epidemic lineages. ${ }^{64-66}$ The emergence of epidemic clones from a background of polyclonality has also been reported in multiple studies applying WGS to regional Sets of $P$. aeruginosa isolates from cystic fibrosis (CF) patients. While it had been doctrine that infections in CF patients are due to acquisition of environmental strains, Dettman and colleagues applied WGS to demonstrate the existence of common strain infecting CF patients in multiple hospitals in North America and the United Kingdom. ${ }^{67}$ Work by this same group and others went on to show that this epidemic lineage had acquired genetic variants beneficial in the CF lung environment and spread globally. ${ }^{68}$

Two recent genomic epidemiological analyses of regional Neisseria gonorrhoeae were among the first to move beyond the description of population structures to identify epidemiological drivers of transmission. ${ }^{69,70}$ By integrating robust epidemiological data on timing of infections, sexual

This article is protected by copyright. All rights reserved. 
preference, and past contacts into phylogenetic analyses, these studies were able to demonstrate local geographic clustering of strains circulating among individuals with common sexual preference. Of note, the study by De Silva et al., which focused on cases from the city of Brighton over a 4-year period, found that local transmission networks in Brighton were supplemented by outside importations from both geographic disparate parts of the United Kingdom as well as the United States. ${ }^{69}$ Thus, by casting an increasingly wider net, the authors were able to determine the relative impact of transmission and importation at different levels of geographic granularity.

\section{(}

These and other genomic epidemiology studies have begun to inform our understanding of the pathways by which MDRO lineages have spread at local and global scales. However, these prior studies have largely been descriptive, in the sense that the clinical and epidemiological factors that affect regional MDRO prevalence and drive the spread of MDROs across healthcare institutions remain largely hidden. For MDROs that primarily spread within healthcare settings, it is likely that patient movement between healthcare facilities drives regional spread. Through the integration of genomic and patient -transfer data, we were able to demonstrate that a handful of patient-transfer events were sufficient to explain a regional outbreak of carbapenem-resistant $K$. pneumoniae that affected 40 patients and 26 healthcare facilities in four adjacent counties in Indiana and Illinois. ${ }^{55}$ Moving forward, it will be important to expand on this proof-of-principle study, overlaying additional metadata on clinical practices and resident patient populations, such that we can understand what drives variation in the prevalence of different MDRO species across healthcare settings, even over short geographic distances. Finally, full understanding of MDRO spread will require more comprehensive sampling. This will require consideration of not only clinical isolates but also isolates gathered through active surveillance culturing of asymptomatic individuals across facilities comprising connected healthcare networks.

This article is protected by copyright. All rights reserved. 


\section{Evolution and dissemination of clonal lineages}

A recurring theme from both genomic and classical molecular epidemiological studies of various MDR Gram-negative organisms is the observation of epidemic lineages that have emerged and spread globally since the dawn of the antibiotic era. ${ }^{71,72}$ The proliferation of these lineages has prompted many investigators to search for common characteristics that might account for their success, but thus far the only characteristic that unifies these epidemic clones is their resistance to one or more common antimicrobials. ${ }^{72}$ hile antibiotic resistance is by definition necessary for a clone to become epidemic, it does not appear in itself sufficient, as there are numerous examples of less prominent clones having the same resistance determinants as their epidemic counterparts. ${ }^{64,73,74}$ Far from being simply an academic exercise, understanding why certain lineages explode on the global scene is critical for more effective monitoring and early detection of emergent lineages of high epidemic potential. By applying evolutionary genomics approaches, investigators have begun to chart the evolutionary trajectories of epidemic lineages, which is an essential first step in understanding whether the suceess of these clones is due to chance accumulation of beneficial mutations or if the genetic background of these ancestral strains predisposed them to thrive in the antibiotic era. Several of these lineages have been reviewed in detail elsewhere, and we therefore provide only a brief discussion of this area. ${ }^{67,72,73,75}$

As alluded to above, antibiotic resistance is a common feature of epidemic clones that have emerged in the antibiotic era. However, the critical resistance determinants and the mode by which they were acquired vary among MDRO lineages. ST131 is a globally disseminated clone of Escherichia coli that is associated with both community- and healthcare-acquired infections. ${ }^{76}$ ST131 stands out among other $E$. coli lineages because of its common association with fluoroquinolone and $\beta$-lactam resistance, mediated by target site mutations and a plasmid-associated extended-spectrum $\beta$ lactamase (ESBL), respectively. ${ }^{72,76}$ Recent comparative genomics studies have revealed a nested substructure to the ST131 lineage, wherein there was a sequential acquisition of fluoroquinolone

This article is protected by copyright. All rights reserved. 
resistance-conferring mutations, followed by a bla-CTX-M-15-containing plasmid. ${ }^{76,77}$ Further work has unearthed additional complexity, in that the bla-CTX-M-15 gene seems to be found on multiple plasmids, which vary in their cargo, indicating that there may have been multiple plasmid-acquisitions events. ${ }^{76} \mathrm{~A}$ second lineage defined by a resistance plasmid is bla-KPC-carrying ST258 Klebsiella pneumoniae. While ST258 can be resistant to nearly all antibiotics, its global proliferation appears to have coincided with the acquisition of a bla-KPC-carrying plasmid. ${ }^{65,78}$ Similar to ST131, bla-KPC has been observed in multiple plasmid contexts within ST258. ${ }^{79}$ It is noteworthy that, while the plasmid backbones vary, both bla-CTX-M-15 and bla-KPC are typically carried on narrow host range IncF plasmids, suggesting that these plasmids may harbor characteristics that either minimize the cost of plasmid maintenance or encode for other features that are beneficial to epidemic clones. ${ }^{80}$

In contrast to the ST131 and ST258 lineages that are defined by the acquisition of particular resistance elements, the European epidemic clones I and II (ECI and ECII) of A. baumannii appear to be defined more by the breadth and flexibility of their resistome. ${ }^{75,81,82}$ While ECI and ECII strains carry resistance plasmids, the defining feature of these strains are massive chromosomally encoded resistance islands that contain multiple antibiotic-resistance determinants that are associated with mobile genetic elements. ${ }^{83-85}$ These resistance islands have proved to be extremely dynamic, with many different configurations reported. ${ }^{86}$ In addition to horizontally acquired elements, $A$. baumannii also has several intrinsic resistance genes, including $\beta$-lactamases and efflux pumps, which can become activated under antibiotic pressure by mobilization of IS elements that carry strong promoters. ${ }^{84,87}$ Similar to A. baumannii, resistance in MDR epidemic clones ST235 and ST111 of $P$. aeruginosa is also attributable to a combination of intrinsic resistance elements and chromosomally associated mobile elements. ${ }^{88}$

While most attention has been given to acquisition of antibiotic-resistance determinants, several MDRO epidemic lineages have also acquired foreign genetic material with the potential to confer advantages beyond survival under antibiotic pressure. Recent comparative genomic studies of

This article is protected by copyright. All rights reserved. 
K. pneumoniae ST258 found that among the few defining events in the emergence of this lineage were two large recombination events that resulted in altered capsular biosynthetic loci. ${ }^{65,79}$ It has been hypothesized that these capsular switching events are important for immune evasion and persistence in hosts. Recombination events altering antigenic molecules have also been observed in E. coli and $A$. baumannii, with recombinant switching of lipopolysaccharide (LPS) loci reported in both species. ${ }^{28,8990}$ In addition to altering putative antigenic determinants, horizontal transfer events have also been observed that have the potential to modify interactions with the host environment in other ways. Studies in ST131 and ST258 have both found horizontal transfer events affecting fimbriae and pili, which may provide advantages in host colonization. ${ }^{65,91,92}$

Despite large comparative genomic studies charting the evolutionary trajectories of prominent Gram-negative lineages, it still remains unclear what has made these epidemic clones so successful. One fundamental question is whether the acquisition of key resistant determinants were the critical events that propelled these lineages or if instead it was the genetic background of the ancestors of epidemic clones that primed them for success. To begin to address this question will require a better understanding of capabilities of these organisms outside of their resistance. For example, studies that assess alternate explanations for success, such as environmental heartiness, capacity for efficient colonization of the host, and the ability to outcompete resident microbiota, are needed to identify factors that underlie the success of these lineages. ${ }^{93}$ A second issue hindering our understanding of the emergence of epidemic lineages is a potential observation bias, wherein the dissemination and evolution of resistant organisms is preferentially monitored compared with their susceptible counterparts. More comprehensive surveillance sampling of organisms regardless of resistance or virulence phenotypes would enable generation of a more complete picture of the global population structure of prevalent pathogens with epidemic lineages and facilitate the retracing of temporal events leading up to the emergence of new dominant resistant lineages. Another form of observation bias is the preferential sampling of individuals in healthcare settings, despite the existence of both resistant

This article is protected by copyright. All rights reserved. 
and susceptible strains of MDRO species circulating in the community. This makes it unclear whether these strains were previously spreading effectively outside hospitals or if the prevalence of these lineages exploded due to acquisition of resistance and selection in the high-antibiotic environment of healthcare facilities. Once we begin to understand the basis for success of dominant resistant lineages, we may be able recognize and predict the emergence of resistant organisms, with the goal of intervening before they negatively affect public health.

\section{Evolution of antibiotic resistance}

While epidemic lineages are of special interest due to their prevalence and tendency towards multidrug resistance, the evolution and spread of antibiotic resistance in less-prolific Gram-negative lineages is atso of major concern for several reasons. First, increased resistance is expected to overall be associated with worse patient outcomes due to increased time to optimal therapy. ${ }^{94}$ Second, resistanee determinants in low-risk clones can become mobilized and be transferred to other MDRO lineages and to other species. ${ }^{95,96}$ Finally, as discussed above, it is unclear if and when the acquisition of a resistant determinant in a low-risk clone could set it on a trajectory toward becoming a significant regional orglobal threat. In recent years, bacterial genomics has been applied to track the real-time evolution of resistance within patients, to elucidate genetic mechanisms underlying resistance in different MDROs, and to characterize the relationship between antibiotic resistance determinants found in different human and environmental reservoirs. ${ }^{97-100}$ It is hoped that these insights into the evolution and ecology of antibiotic resistance can motivate the conception and implementation of more effective strategies for impeding the proliferation of resistance.

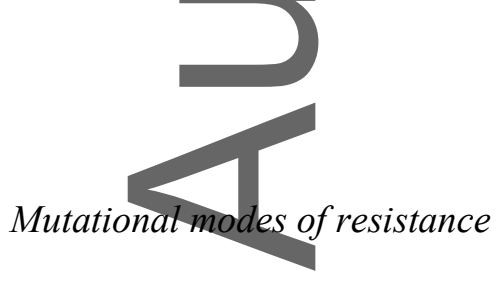

This article is protected by copyright. All rights reserved. 
The most straightforward experimental design for studying clinical resistance evolution is the application of WGS to longitudinal isolates taken from patients in which resistance has evolved during the course of treatment. In these situations, it is presumed that, if resistance has emerged during a short treatment course, it is likely due to a small number of high-impact mutations. ${ }^{97}$ Indeed, studies employing this approach to study resistance evolution typically only observe a handful of mutations between intrapatient pairs, which facilitates the identification of causal variants by identifying genes or pathways mutated in multiple patient time courses. ${ }^{98} \mathrm{~A}$ drug for which several groups have studied intrapatient resistance evolution is colistin. Colistin is a last-line drug for treating MDR Gram-negatives that are resistant to carbapenem antibiotics. ${ }^{101,102}$ The prospect of widespread colistin resistance is of great concern, as there are limited treatment options beyond colistin for the treatment of infeetions caused by carbapenemase-producing Gram-negatives, such as K. pneumoniae and A. baumannii. ${ }^{103}$ Interestingly, in both K. pneumoniae and A. baumannii, genomic sequencing studies have found mutations in a common regulatory pathway controlling LPS modification systems, indicating that LPS modification is key to resistance in both species. ${ }^{104-106}$ This genome-derived hypothesis that altered LPS modification underlies resistance was ultimately confirmed for both organisms by comparing LPS modifications in susceptible and resistant isolates. ${ }^{104,107}$

An important caveat in studying resistance evolution in individual patients is that the larger epidemiological significance of observed resistance mutations or mechanisms cannot necessarily be inferred. This disconnect between short-term and long-term impacts of resistance evolution is due to fitness costs associated with resistance that might limit the ultimate viability of resistant mutants once the selective pressure of the drug is removed. ${ }^{108}$ In other words, resistance alleles that emerge within patients might not be sufficiently fit to effectively spread to other patients. To understand the fate of colistin-resistant mutants in A. baumannii, we collected additional patient isolates following withdrawal of colistin treatment and found that the fitness cost associated with resistance was so severe that, soon after colistin was withdrawn, susceptible isolates re-emerged and outcompeted

This article is protected by copyright. All rights reserved. 
resistant isolates within individual patients. ${ }^{28}$ However, in one patient, we ultimately identified a lowcost resistance mutant that emerged and was sufficiently fit to be detected following termination of colistin treatment. We then went on to show that this mutant was transmitted to other patients, thereby providing additional evidence for its relative fitness and its potential to be a resistance mutant with epidemic potential.

Another important consideration in studying resistance evolution in patients is that there can be multiple resistance alleles present in infecting populations, which will be missed if WGS is performed on only a few clones. ${ }^{21}$ Moreover, it may not always be obvious from which colonizing or infecting population within the patient resistance emerged. For example, many MDROs initially colonize the gastrointestinal tract before migrating and causing infections at other sites, such as the lungs or blood. ${ }^{109,110}$ If a patient has sequential susceptible and resistant isolates taken from their lung, it could be that resistant isolates actually emerged in the GI tract and migrated back to the lung. This distinction may ultimately be extremely important in understanding the population dynamics underlying resistanee evolution and gaining a better understanding of the probability of resistance emergence during treatment.

\section{(rorizontal tyenserand \\ Horizontal transfer and acquisition of resistance} In contrast to the large number of studies documenting mutational resistance emergence in patients, there have been fewer reports documenting horizontal gene transfer (HGT) underlying resistance emergence during treatment. The difficulty in studying HGT derives from the fact that these are presumed to be rare events, and because it can be difficult to demonstrate that the transfer event occurred in a patient, even when the putative source and donor strains are isolated. Despite these challenges, anecdotal reports have begun to emerge documenting the transfer of resistance within the context of individual patients. ${ }^{11,112}$ Through a combination of experimental and clinical evidence,

This article is protected by copyright. All rights reserved. 
Sidjabat et al. demonstrated that, in a single patient, the KPC gene was likely transferred from an infecting K. pneumoniae to E. coli via recombination of plasmid sequences and then subsequently transferred to Serratia marcescens via conjugation. ${ }^{111}$ Hardiman et al. attempted to understand drivers of resistance transfer in patients by measuring in vitro KPC transfer rates with different plasmid backgrounds and environmental conditions. ${ }^{113}$ However, in this study, in vitro conjugation rates did not correlate with presumed in vivo rates of plasmid mobilization in patients during an outbreak, highlighting the need for future studies that determine factors associated with horizontal transfer during patient treatment.

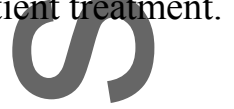

The observation that the transfer of resistance elements between different MDRO strains and species may not be as uncommon as once thought has led investigators to attempt to track the spread of resistance proliferation in the context of hospital outbreaks. ${ }^{114,115}$ For example, the outbreak investigated by Mathers et al. revealed that the prevalence of CRE at this institution was due to a highly complex plasmid transfer network, where intergenus transfer of a promiscuous KPC plasmid, transposition of KPC onto different plasmids backbones, and circulation of diverse $\mathrm{KPC}^{+}$lineages all manifested during the CRE outbreak. Similarly, Conlan et al. identified both shared and distinct carbapenemase-carrying plasmids in several Enterobacteriacae species at their institution. Adding to this complexity, the authors found that, while in some cases intrapatient horizontal transfer of resistance elements was likely, in other cases patients harbored multiple species with common resistance elements where HGT was clearly not the origin. Both studies highlight the complex pathways by which mobile resistance elements spread and the importance of not only monitoring prevalence of resistance but also tracking the mobile genetic elements capable of disseminating resistance in healthcare settings.

In additionto resistance transfer between MDRO species within patients, it is thought that other reservoirs within and outside hospitals may be hubs for resistance dissemination. ${ }^{116,117}$ One potential reservoir of resistance makers outside of healthcare settings is hospital effluent. Several

This article is protected by copyright. All rights reserved. 
groups have used metagenomics approaches to detect resistance markers and mobile genetic elements in hospital wastewater. ${ }^{118-122}$ Growing evidence that environmental water organisms can take up clinically important resistance markers when exposed to these wastewaters further bolsters this hypothesis. ${ }^{122}$ Recently, Rowe et al. used a combined metagenomic/metatranscriptomic approach to measure resistance gene abundance and expression, as well as antibiotic concentrations in effluents from different sites that varied in antibiotic use. ${ }^{120}$ In support of hospital practices playing a role in promoting the environmental resistome, they found that catchment water from hospitals was enriched for $\beta$-lactamases compared with other sites and that hospital effluent $\beta$-lactamase levels correlated with hospital antibiotic usage over time. ${ }^{120}$ Water sources within the hospital have also been implicated as a location where resistance transfer could occur. Recent work investigating the role of sinks, drains and other hospital waterways are motivated by several reports of outbreaks where resistant organisms have been isolated from these sites. ${ }^{123}$

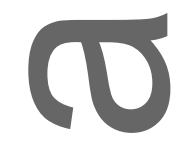

The debate over the relative contributions of different reservoirs to resistance dissemination within hospitals recently came to a head in the case of $m c r-1$, which confers transferrable colistin resistance. Since its initial observation on an inter-species plasmid in 2015, $\mathrm{mcr}-1$ has been identified in the human gut microbiome, wastewater, community, and animal sources. ${ }^{124-128}$ This identification of a previously unknown mobile resistance element in all previously mentioned hypothesized reservoirs demonstrates for $m c r-1$ what is likely true for other resistance mechanisms: that the transfer and dissemination of mobile resistance is likely due to a complex chain of events that take place across multiple ecological settings. Much of the controversy over which reservoirs are the most important for breeding resistance in healthcare settings stems from the fact that the definitive studies examining relative contributions of hypothesized reservoirs of resistance for prevalent pathogens have yet to be carried out. The reservoir is likely different for different pathogens, given that the natural histories of various resistant organisms (e.g., environmental heartiness, colonization niche) differ

This article is protected by copyright. All rights reserved. 
significantly. Further complicating the elucidation of the role of hypothesized resistance reservoirs is that the detection of a resistance marker in a particular location does not inform the timing or direction of resistance transfer from one putative reservoir to another.

Though it is clear that horizontal transfer of resistance is important to the epidemiology of resistant Gram-negatives, there are several fundamental unanswered questions regarding the mechanism and pathways of resistance transfer. For example, though there is extensive in vitro work examining the fitness effects of mutations contributing to antibiotic resistance, the fitness costs of carrying particular resistance elements or mutations in the context of hypothesized real-world reservoirs and patient carriage or infection are unknown. ${ }^{108}$ Studies that examine the evolution of organisms within their real-world context (e.g., longitudinal sampling of colonized patients, evolution on colonized hospital surfaces) are needed to address these questions. Furthermore, little is known about where resistance initially emerges and what clinical and environmental risk factors drive emergence. For example, while multiple studies have assessed the impact of targeted infection preventatives aimed at decolonization of patients or water reservoirs independently, studies that measure the retative contribution of multiple reservoirs, as well as the cost effectiveness and efficacy of different decontamination strategies on patient outcomes, are needed. ${ }^{129-132}$ Lastly, while it is known that particular patients with specific characteristics are more prone to developing resistant infections, it has yet to be assessed how the risk of having resistance emerge through mutation or transfer during the context of particular antibiotic treatments is distributed among patients. So far, studies of resistance evolution in patients have been predominantly anecdotal, and therefore there is little insight into why resistance emerges in particular patients and not in others.

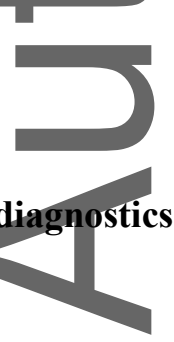

This article is protected by copyright. All rights reserved. 
A central objective of the clinical microbiology laboratory is to gather information about the causative organism of an infection in order to guide optimal therapy. ${ }^{133,134}$ Rapid organism identification is critically important, as delays in the initiation of appropriate treatment are associated with poor patient outcomes. This urgent need has led to the deployment of technologies and workflows aimed at reducingturnaround times between sample collection, organism identification, and susceptibility testing. ${ }^{26,13}$ Newer rapid-identification methods, such as MALDI-ToF, have drastically reduced turnaround time in clinical microbiology laboratories and led to improvements in empiric antibiotic prescribing practices. ${ }^{137-139}$ However, while these rapid-identification methods have yielded some gains, they are often limited in their speed by the need to first culture specimens and limited in their utility by only proyiding species-level classifications. Strain-level discrimination is not only vital information for infection control teams in their efforts to determine if an outbreak is occurring, but could also be utilized in guiding treatment decisions, as certain lineages have the strong associations with resistance and virulence phenotypes. ${ }^{140-144}$ Despite our incomplete understanding of the genetic mechanisms of virulence and resistance for prominent pathogens, genome-guided methods hold great promise as rapid clinical diagnostics with the potential to reduce turnaround times for organism identification and susceptibility testing, as well as aid infection-prevention investigations, by providing the ultimate resolution for determining relatedness of strains in healthcare settings.

Reliance on microbial culture hinders rapid organism identification because culture can take days, or in some cases weeks, for some resistant organisms. ${ }^{57,145}$ For this reason, much attention has shifted to development of culture-free diagnostics, which have the ability to identify the causative agent of infections and outbreaks of unknown etiology. ${ }^{146-149}$ For example, a recent study applied metagenomic sequencing directly to prosthetic joint infection samples and demonstrated that this technique can be used to accurately diagnose this type of infection. ${ }^{150}$ The application of genomics directly to patient samples is particularly attractive for prosthetic joint infections, as the organisms that cause these infections can be present at very low levels and take 1-2 weeks to grow, whereas

This article is protected by copyright. All rights reserved. 
genomic pipelines can detect organisms and identify clinically relevant phenotypes within hours. ${ }^{151-154}$ The management of another slow-growing organism for which resistance is a concern, Mycobacterium tuberculosis, is another clinical situation that might be improved with rapid genomic diagnostics. Votintseva et al. recently showed highly accurate identification and susceptibility profiling of clinical $M$. tuberculosis samples in $\sim 12 \mathrm{~h}$, whereas culture-dependent phenotypic methods for M. tuberculosis susceptibility profiling can take weeks to months. ${ }^{154}$ The outcomes of critically ill patients depend on how fast they can start appropriate antibiotic therapy; however, typical turnaround times for susceptibility testing in clinical microbiology laboratories for even non-slowgrowing organisns range from 2-3 days to weeks, which may be detrimentally long in certain cases. ${ }^{57,155}$ Further illustrating the potential for rapid turnaround, Leggett et al. recently demonstrated real-time organism and resistance profile identification from the feces of an ill infant that took less than 1 hour. 53

Before an organism has been identified in a clinical diagnostic laboratory, patients with severe infections (e.g., bloodstream infections) are often treated empirically, and they are then switched to an antibiotic with known efficacy once a pathogen and its susceptibilities are determined. ${ }^{156}$ In addition to organism identification, genomic methods have the potential to decrease time-to-appropriate-therapy initiation by identifying resistance markers and virulence factors. Culture-free susceptibility identification methods are particularly attractive, as the antibiotic susceptibility of the infecting organism is also major barrier to the rapid initiation of appropriate and successful treatment. ${ }^{26,57,94,157,158}$ There are now several platforms that can be used to predict resistance phenotypes from WGS data. ${ }^{159,160}$ In addition to susceptibilities, information from the genomic detection of virulence determinants could aid in the identification of high-risk strains, providing insight into the probable disease they may cause or their transmissibility in hospital settings. ${ }^{161,162}$ For example, the epidemic lineage $\mathrm{KPC}^{+} K$. pneumoniae ST258 shows variation in its virulence depending on the KPC allele it carries and is hypothesized to be more virulent because of genetic

This article is protected by copyright. All rights reserved. 
changes in its capsule locus. ${ }^{163}$ Rapid identification of organisms belonging to hypervirulent or highly transmissible clones could alert healthcare practitioners to place these patients into appropriate infection control precautions and rapidly initiate effective therapy.

In addition to the application of genomic diagnostics to guide patient treatment, there is interest in the use of genomics in clinical laboratories for surveillance and outbreak detection. Several genomically informed rapid-typing methods have been developed. For example, a genomically informed approach to design multiplex PCR assays that was recently developed has the potential to rapidly identify causative agents in polymicrobial infections as well as identify relatedness between strains in outbreak settings. ${ }^{164,165}$ This application is particularly appealing because, though it is informed by WGS data, its use does not require the operator to have the skill set required to analyze genomic data, which is an additional concern to implementing genomic diagnostics into clinical microbiology workflows. ${ }^{164,166}$ Furthermore, genomics can be used as a gold standard to validate or refute user-friendly typing schemes that are commonly used. A recent study devised a new MLST typing method for Salmonella and validated this method against a core genome phylogeny to demonstrate its utility in distinguishing strains. ${ }^{167}$
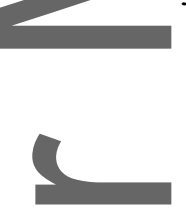

Despite advances in genomic diagnostics, there is so far only one example in the literature of a patient outcome being improved by genomics in real time. ${ }^{168}$ High-risk and time-sensitive infections, such as those in immune-compromised patients or sepsis, could benefit immensely from this technology, but moving real-time genomic diagnostics into clinics and public health labs will overcoming require overcoming several additional hurdles. First, analysis platforms must be adapted for use by personnel in clinieal microbiology laboratories. Second, there have so far been no trials that assess whether patient outcomes improve with implementation of genomic approaches. An attractive application of genomic prediction of resistance or virulence phenotypes is to target decolonization to patients who are colonized, with the goal of reducing their risk for developing and spreading

This article is protected by copyright. All rights reserved. 
infections. Currently, it is unknown whether precision decolonization would prove to be beneficial for patients, or if the benefit would be outweighed by the resulting increase in antibiotic use, which might increase the burden of resistance and put patients at further risk for acquisition of resistant pathogens.

Recent work illustrates both the potential and challenges of the implementation of real-time genomic diagnostics in clinical laboratories. Shelburne et al. demonstrated that WGS accurately predicted resistance to extended-spectrum $\beta$-lactams in major Gram-negative pathogens, suggesting that it may be feasible to use WGS to identify resistance phenotypes in clinical settings. ${ }^{170}$ Still, resistance-prediction methods are limited by their ability to identify known markers, and existence of unknown resistance markers is a major concern for patients, as false susceptibility identification poses a real threat to patient outcomes. If clinical workflows are to move toward phenotype-independent susceptibility prediction, more effective approaches for prediction of unknown resistance genotypes that are scalable for real-time diagnostic workflows are needed. When combined with future methods of improved suseeptibility prediction, a promising technology in the realm of rapid identification and susceptibility testing is the Oxford Nanopore platform. Already, this platform has the capacity to generate sequence data sufficient for species identification in under an hour with computational steps performed on standard laptop computers. ${ }^{153,171,172}$ Whether these new technologies are best applied in everyday diagnestic workflows or reserved for surveillance and outbreak settings remains to be evaluated. Though it is evident that genomic approaches have the potential to revolutionize clinical medicine, unlocking this potential will require key studies that determine whether the cost of implementing these technologies improves patient outcomes.

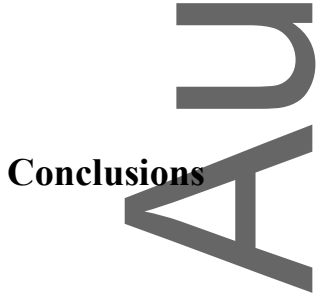

This article is protected by copyright. All rights reserved. 
To summarize, there is an extensive and growing body of work showing how the application of WGS can improve our understanding of the epidemiology and evolution of MDR Gram-negative pathogens. We believe that the next step for moving the field of genomic epidemiology forward is to undertake studies integrating WGS into epidemiologic frameworks from a study's first conception, such that sample collection and analysis methods can be tailored to test specific epidemiologic hypotheses and identify areas where genomics can improve health outcomes. The design and undertaking of these studies is not trivial and will require will require participation from experts across fields, including clinical medicine, microbiology, bioinformatics, antimicrobial stewardship, and healthcare epidemiology. Table 1 illustrates several fundamental questions in healthcare epidemiology, from before genomics were intractable, that are now within reach with the power of genomics and epidemiology combined (Table 1). With continued improvement in sequencing technologies and data analysis strategies, we are on the cusp of fulfilling the promise of genomics to elucidate the practices that drive the emergence and spread of antibiotic resistance, and guide interventions to prevent it. Acknowledgments

SEH was supported by NIH T32-GM113900. SEH and ESS both contributed to the conceptualization, drafting, and editing of this manuscript.

\section{Competing interests}

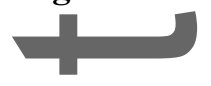

The authors declare no competing interests.

\section{References}

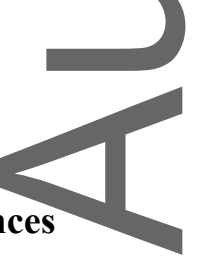

This article is protected by copyright. All rights reserved. 
1. Neu H.C. 1992. The Crisis in Antibiotic Resistance. Science 257: 1064-1073.

2. Chen L. 2017. Notes from the Field: Pan-Resistant New Delhi Metallo-Beta-LactamaseProducing Klebsiella pneumoniae - Washoe County, Nevada, 2016. MMWR Morb. Mortal. Wkly. Rep. 66:33.

3. Lack of development of new antimicrobial drugs: a potential serious threat to public health. Accessed September 16, 2016.

http:/Awww.sciencedirect.com/science/article/pii/S1473309905012831.

4. Spellberg B., R. Guidos, D. Gilbert, et al. 2008. The Epidemic of Antibiotic-Resistant Infections: A Call to Action for the Medical Community from the Infectious Diseases Society of America. Clin. Infect. Dis. 46: 155-164.

5. Vital Signs: Preventing Antibiotic-Resistant Infections in Hospitals - United States, 2014 | MMWR. Accessed September 16, 2016. http:/torigin.glb.cdc.gov/mmwr/volumes/65/wr/mm6509e1.htm.

6. Hidron A.I., J.R. Edwards, J. Patel, et al. 2008. Antimicrobial-Resistant Pathogens Associated With Healthcare-Associated Infections: Annual Summary of Data Reported to the National Healthcare Safety Network at the Centers for Disease Control and Prevention, 2006-2007. Infect. Control Amp Hosp. Epidemiol. 29: 996-1011.

7. Venditti M., M. Falcone, S. Corrao, et al. 2009. Outcomes of Patients Hospitalized With Community-Acquired, Health Care-Associated, and Hospital-Acquired Pneumonia. Ann. Intern. Med.150: 19=26.

8. Cosgrove S.E. 2006. The Relationship between Antimicrobial Resistance and Patient Outcomes: Mortality, Length of Hospital Stay, and Health Care Costs. Clin. Infect. Dis. 42: S82-S89.

9. Ventola C.L. 2015. The Antibiotic Resistance Crisis. Pharm. Ther. 40: 277-283.

10. Bathoorn E, C. Tsioutis, da S. Voorham, et al. 2016. Emergence of pan-resistance in KPC-2 carbapenemase-producing Klebsiella pneumoniae in Crete, Greece: a close call. J. Antimicrob. Chemother. 71: 1207-1212.

11. Sonnevend Á.,A. Ghazawi, R. Hashmey, et al. 2017. Multi-hospital occurrence of pan-resistant Klebsiella pneumoniae ST147 with an ISEcp1-directed blaOXA-181 insertion into the mgrB gene in the United Arab Emirates*. Antimicrob. Agents Chemother. doi: 10.1128/AAC.0041817

12. Tenover F.C., R.D. Arbeit \& R.V. Goering. 1997.Infection Control \&amp; Hospital Epidemiology June 1997 Accessed April 4, 2017. /core/journals/infection-control-and-hospitalepidemiology/article/how-to-select-and-interpret-molecular-strain-typing-methods-forepidemiological-studies-of-bacterial-infections-a-review-for-healthcareepidemiologists/F3D5474D0557355C933590E4C580D3CC.

This article is protected by copyright. All rights reserved. 
13. Tenover F.C., R.D. Arbeit, R.V. Goering, et al. 1995. Interpreting chromosomal DNA restriction patterns produced by pulsed-field gel electrophoresis: criteria for bacterial strain typing. J. Clin. Microbiol. 33: 2233-2239.

14. Maiden M.C.J., J.A. Bygraves, E. Feil, et al. 1998. Multilocus sequence typing: A portable approach to the identification of clones within populations of pathogenic microorganisms. Proc. Natl. Acad. Sci. 95: 3140-3145.

15. Olive D.M. \& P. Bean. 1999. Principles and Applications of Methods for DNA-Based Typing of Microbial Organisms. J. Clin. Microbiol. 37: 1661-1669.

16. Köser C.U., M.T.G. Holden, M.J. Ellington, et al. 2012. Rapid Whole-Genome Sequencing for Investigation of a Neonatal MRSA Outbreak. N. Engl. J. Med. 366: 2267-2275.

17. Snitkin E.S., A.M. Zelazny, P.J. Thomas, et al. 2012. Tracking a Hospital Outbreak of Carbapenen-Resistant Klebsiella pneumoniae with Whole-Genome Sequencing. Sci. Transl. Med.4: 148ral 16-148ra116.

18. Chin C.-S., J.Sorenson, J.B. Harris, et al. 2011. The Origin of the Haitian Cholera Outbreak Strain. N. Engl. J. Med. 364: 33-42.

19. Eyre D.W., M.L. Cule, D.J. Wilson, et al. 2013. Diverse Sources of C. difficile Infection Identified on-Whole-Genome Sequencing. N. Engl. J. Med. 369: 1195-1205.

20. Roetzer A., R.Diel, T.A. Kohl, et al. 2013. Whole Genome Sequencing versus Traditional Genotyping for Investigation of a Mycobacterium tuberculosis Outbreak: A Longitudinal Molecular Epidemiological Study. PLOS Med. 10: e1001387.

21. DidelotX., A.S. Walker, T.E. Peto, et al. 2016. Within-host evolution of bacterial pathogens. Nat. Rev. Microbiol. 14: 150-162.

22. KEGG: Kyoto Encyclopedia of Genes and Genomes. Accessed November 22, 2017. http://www.genome.jp/kegg/.

23. Tatusor R.L., M.Y. Galperin, D.A. Natale, et al. 2000. The COG database: a tool for genomescale analysis of protein functions and evolution. Nucleic Acids Res. 28: 33-36.

24. Wattan A.R., D. Abraham, O. Dalay, et al. 2014. PATRIC, the bacterial bioinformatics database and analysis resource. Nucleic Acids Res. 42: D581-D591.

25. Giske C.G., D.L. Monnet, O. Cars, et al. 2008. Clinical and Economic Impact of Common Multidrug-Resistant Gram-Negative Bacilli. Antimicrob. Agents Chemother. 52: 813-821.

26. Kang C.-I., S.-H. Kim, W.B. Park, et al. 2005. Bloodstream Infections Caused by AntibioticResistant Gram-Negative Bacilli: Risk Factors for Mortality and Impact of Inappropriate Initial Antimicrobial Therapy on Outcome. Antimicrob. Agents Chemother. 49: 760-766.

This article is protected by copyright. All rights reserved. 
27. Lewis T., N.J. Loman, L. Bingle, et al. 2010. High-throughput whole-genome sequencing to dissect the epidemiology of Acinetobacter baumannii isolates from a hospital outbreak. J. Hosp. Infect. 75: 37-41.

28. Snitkin E.S., A.M. Zelazny, C.I. Montero, et al. 2011. Genome-wide recombination drives diversification of epidemic strains of Acinetobacter baumannii. Proc. Natl. Acad. Sci. 108: $13758-13763$.

29. Willems S., S. Kampmeier, S. Bletz, et al. 2016. Whole-Genome Sequencing Elucidates Epidemiology of Nosocomial Clusters of Acinetobacter baumannii. J. Clin. Microbiol. 54: $2391-2394$.

30. Kanamori H., C.M. Parobek, D.J. Weber, et al. 2016. Next-Generation Sequencing and Comparative Analysis of Sequential Outbreaks Caused by Multidrug-Resistant Acinetobacter baumannii at a Large Academic Burn Center. Antimicrob. Agents Chemother. 60: 1249-1257.

31. Stoesser N., A.E. Sheppard, M. Shakya, et al. 2015. Dynamics of MDR Enterobacter cloacae outbreaks in a neonatal unit in Nepal: insights using wider sampling frames and next-generation sequencing. J. Antimicrob. Chemother. 70: 1008-1015.

32. Mathers A.J., N. Stoesser, A.E. Sheppard, et al. 2015. Klebsiella pneumoniae Carbapenemase (KPC)-Produeing K. pneumoniae at a Single Institution: Insights into Endemicity from WholeGenome Sequencing. Antimicrob. Agents Chemother. 59: 1656-1663.

33. Halachev M.R., J.Z.-M. Chan, C.I. Constantinidou, et al. 2014. Genomic epidemiology of a protracted hospital outbreak caused by multidrug-resistant Acinetobacter baumanniiin Birmingham, England. Genome Med. 6: 70.

34. Pittet-D., S. Dharan, S. Touveneau, et al. 1999. Bacterial Contamination of the Hands of Hospital Staff During Routine Patient Care. Arch. Intern. Med. 159: 821-826.

35. Pittet D. B. Allegranzi, H. Sax, et al. 2006. Evidence-based model for hand transmission during patient care and the role of improved practices. Lancet Infect. Dis. 6: 641-652.

36. Willmann M.,D. Bezdan, L. Zapata, et al. 2015. Analysis of a long-term outbreak of XDR Pseudomonas aeruginosa: a molecular epidemiological study. J. Antimicrob. Chemother. 70: $1322-1330$.

37. Davis R.J., S.O. Jensen, S.V. Hal, et al. 2015. Whole Genome Sequencing in Real-Time Investigation and Management of a Pseudomonas aeruginosa Outbreak on a Neonatal Intensive Care Unit. Infect. Control Amp Hosp. Epidemiol. 36: 1058-1064.

38. Conlan S., M.Park, C. Deming, et al. 2016. Plasmid Dynamics in KPC-Positive Klebsiella pneumoniae during Long-Term Patient Colonization. mBio 7: e00742-16.

39. O'Fatton E., S. Gautam \& E.M.C. D'Agata. 2009. Colonization with Multidrug-Resistant GramNegative Bacteria: Prolonged Duration and Frequent Cocolonization. Clin. Infect. Dis. 48: $1375-1381$.

This article is protected by copyright. All rights reserved. 
40. Lin I.-W., C.-Y. Huang, S.-C. Pan, et al. 2017. Duration of colonization with and risk factors for prolonged carriage of multidrug resistant organisms among residents in long-term care facilities. Antimicrob. Resist. Infect. Control 6: 86.

41. Haverkate M.R., S. Weiner, K. Lolans, et al. 2016. Duration of Colonization With Klebsiella pneumoniae Carbapenemase-Producing Bacteria at Long-Term Acute Care Hospitals in Chicago, Illinois. Open Forum Infect. Dis. 3: ofw178.

42. Zimmerman F.S., M.V. Assous, T. Bdolah-Abram, et al. 2013. Duration of carriage of carbapenem-resistant Enterobacteriaceae following hospital discharge. Am. J. Infect. Control 41: $190-194$.

43. Roach D.J., J.N. Burton, C. Lee, et al. 2015. A Year of Infection in the Intensive Care Unit: Prospeetive Whole Genome Sequencing of Bacterial Clinical Isolates Reveals Cryptic Transmissions and Novel Microbiota. PLoS Genet 11: e1005413.

44. Stoesser N., A.E. Sheppard, C.E. Moore, et al. 2015. Extensive within-host diversity in fecally carried extended-spectrum beta-lactamase-producing Escherichia coli: implications for transmission analyses. J. Clin. Microbiol. JCM.00378-15.

45. Paterson G.K., E.M. Harrison, G.G.R. Murray, et al. 2015. Capturing the cloud of diversity reveats complexity and heterogeneity of MRSA carriage, infection and transmission. Nat. Commun. 6: 6560 .

46. Maio N.D., C.-H. Wu \& D.J. Wilson. 2016. SCOTTI: Efficient Reconstruction of Transmission within Outbreaks with the Structured Coalescent. PLOS Comput. Biol. 12: e1005130.

47. Didelot X. C.Fraser, J. Gardy, et al. 2017. Genomic Infectious Disease Epidemiology in Partially Sampled and Ongoing Outbreaks. Mol. Biol. Evol. 34: 997-1007.

48. Lieberman T.D., K.B. Flett, I. Yelin, et al. 2013. Genetic variation of a bacterial pathogen within individuals with cystic fibrosis provides a record of selective pressures. Nat. Genet. 46: ng.2848.

49. Walker T.M., C.L. Ip, R.H. Harrell, et al. 2013. Whole-genome sequencing to delineate Mycobacterium tuberculosis outbreaks: a retrospective observational study. Lancet Infect. Dis. 13: $137-146$.

50. Jolley K.A. \& M.C.J. Maiden. 2013. Automated extraction of typing information for bacterial pathogens from whole genome sequence data: Neisseria meningitidis as an exemplar. Euro Surveill. Bull. Eur. Sur Mal. Transm. Eur. Commun. Dis. Bull. 18: 20379.

51. Dekker J.P. \& K.M. Frank. 2016. Next-Generation Epidemiology: Using Real-Time Core Genome Multilocus Sequence Typing To Support Infection Control Policy. J. Clin. Microbiol. 54: $2850-2853$.

52. Harris S.R., E.J. Cartwright, M.E. Török, et al. 2013. Whole-genome sequencing for analysis of an outbreak of meticillin-resistant Staphylococcus aureus: a descriptive study. Lancet Infect. Dis. 13: 130-136.

This article is protected by copyright. All rights reserved. 
53. Worby C.J., M. Lipsitch \& W.P. Hanage. 2014. Within-Host Bacterial Diversity Hinders Accurate Reconstruction of Transmission Networks from Genomic Distance Data. PLoS Comput Biol 10: e1003549.

54. Worby C.J., M. Lipsitch \& W.P. Hanage. 2017. Shared Genomic Variants: Identification of Transmission Routes Using Pathogen Deep-Sequence Data. Am. J. Epidemiol. 186: 1209-1216.

55. Snitkin E.S., S. Won, A. Pirani, et al. 2017. Integrated genomic and interfacility patient-transfer data reveal the transmission pathways of multidrug-resistant Klebsiella pneumoniae in a regional outbreak. Sci. Transl. Med. 9: eaan0093.

56. Moradigaravand D., V. Martin, S.J. Peacock, et al. 2017. Evolution and Epidemiology of Multidrug-Resistant Klebsiella pneumoniae in the United Kingdom and Ireland. mBio 8: e01976-16.

57. Didelot X., R. Bowden, D.J. Wilson, et al. 2012. Transforming clinical microbiology with bacterial genome sequencing. Nat. Rev. Genet. 13: 601-612.

58. Mellmann A., S. Bletz, T. Böking, et al. 2016. Real-Time Genome Sequencing of Resistant Bacteria Provides Precision Infection Control in an Institutional Setting. J. Clin. Microbiol. JCM.00790-16.

59. Ciccolini M., T. Donker, R. Köck, et al. 2013. Infection prevention in a connected world: The case for a regional approach. Int. J. Med. Microbiol. 303: 380-387.

60. Pecora N.D., N Li, M. Allard, et al. 2015. Genomically Informed Surveillance for CarbapenemResistant Enterobacteriaceae in a Health Care System. mBio 6: e01030-15.

61. Moradigaravand D., S. Reuter, V. Martin, et al. 2016. The dissemination of multidrug-resistant Enterobacter cloacae throughout the UK and Ireland. Nat. Microbiol. 1: 16173.

62. Moradigaravand D., C.J. Boinett, V. Martin, et al. 2016. Recent independent emergence of multiple multidrug-resistant Serratia marcescens clones within the United Kingdom and Ireland. Genome Res. 26: 1101-1109.

63. Hargreaves M.L., K.M. Shaw, G. Dobbins, et al. 2015. Clonal dissemination of Enterobacter cloacae harboring blaKPC-3 in the upper midwestern United States. Antimicrob. Agents Chemother. 59:7723-34.

64. Bowers J.R., B. Kitchel, E.M. Driebe, et al. 2015. Genomic Analysis of the Emergence and Rapid Global Dissemination of the Clonal Group 258 Klebsiella pneumoniae Pandemic. PLOS ONE 10: e0133727

65. Chen L., B. Mathema, J.D.D. Pitout, et al. 2014. Epidemic Klebsiella pneumoniae ST258 Is a Hybrid Strain. mBio 5: e01355-14.

66. Zarrilli R., S. Pournaras, M. Giannouli, et al. 2013. Global evolution of multidrug-resistant Acinetobacter baumannii clonal lineages. Int. J. Antimicrob. Agents 41: 11-19.

This article is protected by copyright. All rights reserved. 
67. Dettman J.R., N. Rodrigue, S.D. Aaron, et al. 2013. Evolutionary genomics of epidemic and nonepidemic strains of Pseudomonas aeruginosa. Proc. Natl. Acad. Sci. 110: 21065-21070.

68. Fothergill J.L., M.J. Walshaw \& C. Winstanley. 2012. Transmissible strains of Pseudomonas aeruginosa in cystic fibrosis lung infections. Eur. Respir. J. 40: 227-238.

69. De Silva D., J. Peters, K. Cole, et al. Whole-genome sequencing to determine transmission of Neisseria gonorrhoeae: an observational study. Lancet Infect. Dis. 16:1295-1303

70. Grad Y.H., R.D. Kirkcaldy, D. Trees, et al. 2014. Genomic epidemiology of Neisseria gonorrhoeae with reduced susceptibility to cefixime in the USA: a retrospective observational study. Lancet Infect. Dis. 14: 220-226.

71. Davies J. \&D. Davies. 2010. Origins and Evolution of Antibiotic Resistance. Microbiol. Mol. Biol. Rev. 74: 417-433.

72. Woodford N., J.F. Turton \& D.M. Livermore. 2011. Multiresistant Gram-negative bacteria: the role of high-risk clones in the dissemination of antibiotic resistance. FEMS Microbiol. Rev. 35: $736-755$

73. Kitchel B., J.K. Rasheed, J.B. Patel, et al. 2009. Molecular Epidemiology of KPC-Producing Klebsiella pneumoniae Isolates in the United States: Clonal Expansion of Multilocus Sequence Type 258. Antimicrob. Agents Chemother. 53: 3365-3370.

74. Stoesser N. A.E. Sheppard, G. Peirano, et al. 2017. Genomic epidemiology of global Klebsiella pneumoniae carbapenemase (KPC)-producing Escherichia coli. Sci. Rep. 7:5917.

75. Zarrilli R., S-Pournaras, M. Giannouli, et al. 2013. Global evolution of multidrug-resistant Acinetobacter baumannii clonal lineages. Int. J. Antimicrob. Agents 41: 11-19.

76. Stoesser N., A.E. Sheppard, L. Pankhurst, et al. 2016. Evolutionary History of the Global Emergence of the Escherichia coli Epidemic Clone ST131. mBio 7: e02162-15.

77. Zakour N.L.B., A.S. Alsheikh-Hussain, M.M. Ashcroft, et al. 2016. Sequential Acquisition of Virulence and Fluoroquinolone Resistance Has Shaped the Evolution of Escherichia coli ST131. mBio 7:e00347-16.

78. Bogdanovich T., J.M. Adams-Haduch, G.-B. Tian, et al. 2011. Colistin-Resistant, Klebsiella pneumoniae Carbapenemase (KPC)-Producing Klebsiella pneumoniae Belonging to the International Epidemic Clone ST258. Clin. Infect. Dis. 53: 373-376.

79. DeLeoF.R., L.Chen, S.F. Porcella, et al. 2014. Molecular dissection of the evolution of carbapenem-resistant multilocus sequence type 258 Klebsiella pneumoniae. Proc. Natl. Acad. Sci. 111: 4988-4993.

80. Mathers A.J., G. Peirano \& J.D.D. Pitout. 2015. The Role of Epidemic Resistance Plasmids and International High-Risk Clones in the Spread of Multidrug-Resistant Enterobacteriaceae. Clin. Microbiol. Rev. 28: 565-591.

This article is protected by copyright. All rights reserved. 
81. van Dessel H., L. Dijkshoorn, T. van der Reijden, et al. 2004. Identification of a new geographically widespread multiresistant Acinetobacter baumannii clone from European hospitals. Res. Microbiol. 155: 105-112.

82. Diancourt L., V. Passet, A. Nemec, et al. 2010. The Population Structure of Acinetobacter baumannii: Expanding Multiresistant Clones from an Ancestral Susceptible Genetic Pool. PLOS ONE 5. e10034.

83. WrightM.S., D.H. Haft, D.M. Harkins, et al. 2014. New Insights into Dissemination and Variation of the Health Care-Associated Pathogen Acinetobacter baumannii from Genomic Analysis. mBio 5: e00963-13.

84. Poirel L., R.A Bonnin \& P. Nordmann. 2011. Genetic basis of antibiotic resistance in pathogenic Acinetobacter species. IUBMB Life 63: 1061-1067.

85. Chan A.P., G. Sutton, J. DePew, et al. 2015. A novel method of consensus pan-chromosome assembly and large-scale comparative analysis reveal the highly flexible pan-genome of Acinetobacter baumannii. Genome Biol. 16: 143.

86. Blackwell G.A., S.J. Nigro \& R.M. Hall. 2016. Evolution of AbGRI2-0, the Progenitor of the AbGRI2 Resistance Island in Global Clone 2 of Acinetobacter baumannii. Antimicrob. Agents Chemother. 60: 1421-1429.

87. Figueiredo S., L. Poirel, A. Papa, et al. 2009. Overexpression of the Naturally Occurring blaOXA-51Gene in Acinetobacter baumannii Mediated by Novel Insertion Sequence ISAba9. Antimicrob. Agents Chemother. 53: 4045-4047.

88. Oliver A., X. Mulet, C. López-Causapé, et al. 2015. The increasing threat of Pseudomonas aeruginosa high-risk clones. Drug Resist. Updat. 21: 41-59.

89. Petty N.K., N.L.B. Zakour, M. Stanton-Cook, et al. 2014. Global dissemination of a multidrug resistant Escherichia coli clone. Proc. Natl. Acad. Sci. 111: 5694-5699.

90. Kallonen T.,H.J. Brodrick, S.R. Harris, et al. 2017. Systematic longitudinal survey of invasive Escherichia coli in England demonstrates a stable population structure only transiently disturbed by the emergence of ST131. Genome Res. 27: 1437-1449.

91. Nicolas-Chanoine M.-H., X. Bertrand \& J.-Y. Madec. 2014. Escherichia coli ST131, an Intriguing Clonal Group. Clin. Microbiol. Rev. 27: 543-574.

92. Sarkar S., L.W. Roberts, M.-D. Phan, et al. 2016. Comprehensive analysis of type 1 fimbriae regulation in fimB-null strains from the multidrug resistant Escherichia coli ST131 clone. Mol. Microbiol. 101: 1069-1087.

93. Lebreton F. A.L. Manson, J.T. Saavedra, et al. 2017. Tracing the Enterococci from Paleozoic Origins to the Hospital. Cell 169: 849-861.e13.

This article is protected by copyright. All rights reserved. 
94. Lodise T.P., N. Patel, A. Kwa, et al. 2007. Predictors of 30-Day Mortality among Patients with Pseudomonas aeruginosa Bloodstream Infections: Impact of Delayed Appropriate Antibiotic Selection. Antimicrob. Agents Chemother. 51: 3510-3515.

95. Hall M.A.L., A.T.A. Box, H.E.M. Blok, et al. 2002. Evidence of Extensive Interspecies Transfer of Integron-Mediated Antimicrobial Resistance Genes among Multidrug-Resistant Enterobacteriaceae in a Clinical Setting. J. Infect. Dis. 186: 49-56.

96. Wang X., G. Chen, X. Wu, et al. 2015. Increased prevalence of carbapenem resistant Enterobacteriaceae in hospital setting due to cross-species transmission of the blaNDM-1 element and clonal spread of progenitor resistant strains. Front. Microbiol. 6:595.

97. Silva I.N., P.M. Santos, M.R. Santos, et al. 2016. Long-Term Evolution of Burkholderia multiyorans during a Chronic Cystic Fibrosis Infection Reveals Shifting Forces of Selection. mSystems 1: e00029-16.

98. Marvig R.L., L.M. Sommer, S. Molin, et al. 2015. Convergent evolution and adaptation of Pseudomonas aeruginosa within patients with cystic fibrosis. Nat. Genet. 47: 57-64.

99. Riesenfeld C.S., R.M. Goodman \& J. Handelsman. 2004. Uncultured soil bacteria are a reservoir of new antibiotic resistance genes. Environ. Microbiol. 6: 981-989.

100. McArthur A.G., N. Waglechner, F. Nizam, et al. 2013. The Comprehensive Antibiotic Resistance Database. Antimicrob. Agents Chemother. 57: 3348-3357.

101. Paterson D.L.\& P.N.A. Harris. 2016. Colistin resistance: a major breach in our last line of defence. Lancet Infect. Dis. 16: 132-133.

102. Boucher H.W., G.H. Talbot, J.S. Bradley, et al. 2009. Bad Bugs, No Drugs: No ESKAPE! An Update from the Infectious Diseases Society of America. Clin. Infect. Dis. 48: 1-12.

103. Li J.,(R.L. Nation, J.D. Turnidge, et al. 2006. Colistin: the re-emerging antibiotic for multidrugresistant Gram-negative bacterial infections. Lancet Infect. Dis. 6: 589-601.

104. Leung L.M., V.S. Cooper, D.A. Rasko, et al. 2017. Structural modification of LPS in colistinresistant, KPC-producing Klebsiella pneumoniae. J. Antimicrob. Chemother. 72: 3035-3042.

105. Mu X., N. Wang, X. Li, et al. 2016. The Effect of Colistin Resistance-Associated Mutations on the Fitness of Acinetobacter baumannii. Front. Microbiol. 7:1715.

106. Wright M.S., Y. Suzuki, M.B. Jones, et al. 2015. Genomic and Transcriptomic Analyses of Colistin-Resistant Clinical Isolates of Klebsiella pneumoniae Reveal Multiple Pathways of Resistance. Antimicrob. Agents Chemother. 59: 536-543.

107. Beceiro A., E. Llobet, J. Aranda, et al. 2011. Phosphoethanolamine Modification of Lipid A in Colistin-Resistant Variants of Acinetobacter baumannii Mediated by the pmrAB TwoComponent Regulatory System. Antimicrob. Agents Chemother. 55: 3370-3379.

This article is protected by copyright. All rights reserved. 
108. Melnyk A.H., A. Wong \& R. Kassen. 2015. The fitness costs of antibiotic resistance mutations. Evol. Appl. 8: 273-283.

109. Martin R.M.J. Cao, S. Brisse, et al. 2016. Molecular Epidemiology of Colonizing and Infecting Isolates of Klebsiella pneumoniae. mSphere 1: e00261-16.

110. Gorrie C.L., M. Mirčeta, R.R. Wick, et al. 2017. Gastrointestinal Carriage Is a Major Reservoir of Klebsiella pneumoniae Infection in Intensive Care Patients. Clin. Infect. Dis. 65: 208-215.

111. Sidjabat H.E., F.P. Silveira, B.A. Potoski, et al. 2009. Interspecies Spread of Klebsiella pneumoniae Carbapenemase Gene in a Single Patient. Clin. Infect. Dis. 49: 1736-1738.

112. Goren M.G.,Y. Carmeli, M.J. Schwaber, et al. 2010. Transfer of Carbapenem-Resistant Plasmid from Klebsiella pneumoniae ST258 to Escherichia coli in Patient. Emerg. Infect. Dis. 16: $1014-1017$.

113. Hardiman C.A., R.A. Weingarten, S. Conlan, et al. 2016. Horizontal Transfer of Carbapenemase-Encoding Plasmids and Comparison with Hospital Epidemiology Data. Antimicrob. Agents Chemother. 60: 4910-4919.

114. Mathers A.J., H.L. Cox, B. Kitchel, et al. 2011. Molecular Dissection of an Outbreak of Carbapenem-Resistant Enterobacteriaceae Reveals Intergenus KPC Carbapenemase Transmissionthrough a Promiscuous Plasmid. mBio 2: e00204-11.

115. Conlan S.,P.J. Thomas, C. Deming, et al. 2014. Single-molecule sequencing to track plasmid diversity of hospital-associated carbapenemase-producing Enterobacteriaceae. Sci. Transl. Med. 6: $254 \mathrm{ra1} 26-254 \mathrm{ra} 126$.

116. Wright G.D. 2007. The antibiotic resistome: the nexus of chemical and genetic diversity. Nat. Rev. Microbiol. 5: 175.

117. Allen H.K., H.H. Wang, J. Handelsman, et al. 2010. Call of the wild: antibiotic resistance genes in natural environments. Nat. Rev. Microbiol. 8: 251.

118. Schwartz T., W. Kohnen, B. Jansen, et al. 2003. Detection of antibiotic-resistant bacteria and their resistance genes in wastewater, surface water, and drinking water biofilms. FEMS Microbiol. Ecol. 43: 325-335.

119. Rizzo L., C. Manaia, C. Merlin, et al. 2013. Urban wastewater treatment plants as hotspots for antibiotic resistant bacteria and genes spread into the environment: A review. Sci. Total Environ. 447: $345-360$.

120. Rowe W.P.M., C. Baker-Austin, D.W. Verner-Jeffreys, et al. 2017. Overexpression of antibiotic resistance genes in hospital effluents over time. J. Antimicrob. Chemother. 72: 1617-1623.

121. Breathnach A.S., M.D. Cubbon, R.N. Karunaharan, et al. 2012. Multidrug-resistant Pseudomonas aeruginosa outbreaks in two hospitals: association with contaminated hospital waste-water systems. J. Hosp. Infect. 82: 19-24.

This article is protected by copyright. All rights reserved. 
122. Scotta C., C. Juan, G. Cabot, et al. 2011. Environmental Microbiota Represents a Natural Reservoir for Dissemination of Clinically Relevant Metallo- $\beta$-Lactamases. Antimicrob. Agents Chemother. 55: 5376-5379.

123. Gordon K., A. E, A.J. Mathers, et al. 2017. The Hospital Water Environment as a Reservoir for Carbapenem-Resistant Organisms Causing Hospital-Acquired Infections-A Systematic Review of the Literature. Clin. Infect. Dis. 64: 1435-1444.

124. Liu Y-Y., Y. Wang, T.R. Walsh, et al. Emergence of plasmid-mediated colistin resistance mechanism MCR-1 in animals and human beings in China: a microbiological and molecular biologieal study. Lancet Infect. Dis. 16:161-8.

125. Wintersdorff V., C.J. H, P.F.G. Wolffs, et al. 2016. Detection of the plasmid-mediated colistinresistance gene mcr-1 in faecal metagenomes of Dutch travellers. J. Antimicrob. Chemother. 71: $3416-3419$.

126. Hembach N.,F. Schmid, J. Alexander, et al. 2017. Occurrence of the mcr-1 Colistin Resistance Gene and other Clinically Relevant Antibiotic Resistance Genes in Microbial Populations at Different Municipal Wastewater Treatment Plants in Germany. Front. Microbiol. 8:1282.

127. Doumith M., G. Godbole, P. Ashton, et al. 2016. Detection of the plasmid-mediated mcr-1 gene conferring colistin resistance in human and food isolates of Salmonella enterica and Escherichia coli in England and Wales. J. Antimicrob. Chemother. 71: 2300-2305.

128. Tian G.-B., Y. Doi, J. Shen, et al. 2017. MCR-1-producing Klebsiella pneumoniae outbreak in China. Lancet Infect. Dis. 17: 577.

129. Bar-Yoseph H., K. Hussein, E. Braun, et al. 2016. Natural history and decolonization strategies for ESBL/carbapenem-resistant Enterobacteriaceae carriage: systematic review and metaanalysis. J. Antimicrob. Chemother. 71: 2729-2739.

130. Septimus E.J. \& M.L. Schweizer. 2016. Decolonization in Prevention of Health Care-Associated Infections. Clin. Microbiol. Rev. 29: 201-222.

131. Dancer S.J. 2014. Controlling Hospital-Acquired Infection: Focus on the Role of the Environment and New Technologies for Decontamination. Clin. Microbiol. Rev. 27: 665-690.

132. You J.H.S., H. Li \& M. Ip. Surveillance-guided selective digestive decontamination of carbapenem-resistant Enterobacteriaceae in the intensive care unit: A cost-effectiveness analysis. Am. J. Infect. Control. doi: 10.1016/j.ajic.2017.09.003.

133. Reller L.B., M.P. Weinstein, L.R. Peterson, et al. 2001. Role of Clinical Microbiology Laboratories in the Management and Control of Infectious Diseases and the Delivery of Health Care. Clin. Infect. Dis. 32: 605-610.

134. Emori T.G. \& R.P. Gaynes. 1993. An overview of nosocomial infections, including the role of the microbiology laboratory. Clin. Microbiol. Rev. 6: 428-442.

This article is protected by copyright. All rights reserved. 
135. Harbarth S., J. Garbino, J. Pugin, et al. 2003. Inappropriate initial antimicrobial therapy and its effect on survival in a clinical trial of immunomodulating therapy for severe sepsis. Am. J. Med. 115: $529-535$.

136. Paul M., V. Shani, E. Muchtar, et al. 2010. Systematic Review and Meta-Analysis of the Efficacy of Appropriate Empiric Antibiotic Therapy for Sepsis. Antimicrob. Agents Chemother. 54: $4851-4863$.

137. Dingle T.C. \& S.M. Butler-Wu. 2013. MALDI-TOF Mass Spectrometry for Microorganism Identification. Clin. Lab. Med. 33: 589-609.

138. Cherkaoui A., J. Hibbs, S. Emonet, et al. 2010. Comparison of Two Matrix-Assisted Laser Desorption Ionization-Time of Flight Mass Spectrometry Methods with Conventional Phenotypic Identification for Routine Identification of Bacteria to the Species Level. J. Clin. Microbiol 48: 1169-1175.

139. Clerc O., G. Prod'hom, C. Vogne, et al. 2013. Impact of Matrix-Assisted Laser Desorption Ionization Time-of-Flight Mass Spectrometry on the Clinical Management of Patients With Gram-negative Bacteremia: A Prospective Observational Study. Clin. Infect. Dis. 56: 11011107

140. Vlek A.L.M., M.J.M. Bonten \& C.H.E. Boel. 2012. Direct Matrix-Assisted Laser Desorption Ionization Time-of-Flight Mass Spectrometry Improves Appropriateness of Antibiotic Treatment of Bacteremia. PLOS ONE 7: e32589.

141. Veen S.Q. van, E.C.J. Claas \& E.J. Kuijper. 2010. High-Throughput Identification of Bacteria and Yeast by Matrix-Assisted Laser Desorption Ionization-Time of Flight Mass Spectrometry in Conventional Medical Microbiology Laboratories. J. Clin. Microbiol. 48: 900-907.

142. Sanguinetti M. \& B. Posteraro. 2016. Mass spectrometry applications in microbiology beyond microbe identification: progress and potential. Expert Rev. Proteomics

143. Safdar N., S. Sengupta, J.S. Musuuza, et al. 2016. Status of the Prevention of MultidrugResistant Organisms in International Settings: A Survey of the Society for Healthcare Epidemiology of America Research Network. Infect. Control Amp Hosp. Epidemiol. 38:53-60.

144. Sabat A.J., A. Budimir, D. Nashev, et al. 2013. Overview of molecular typing methods for outbreak detection and epidemiological surveillance. Eurosurveillance 18: 20380.

145. Bertelli C. \& G. Greub. 2013. Rapid bacterial genome sequencing: methods and applications in clinical microbiology. Clin. Microbiol. Infect. 19: 803-813.

146. Seth-Smith H.M.B., S.R. Harris, R.J. Skilton, et al. 2013. Whole-genome sequences of Chlamydia trachomatis directly from clinical samples without culture. Genome Res. 23: 855866

147. A Culture-Independent Sequence-Based Metagenomics Approach to the Investigation of an Outbreak of Shiga-Toxigenic Escherichia coli O104:H4 | Apr 10, 2013 | JAMA | JAMA

This article is protected by copyright. All rights reserved. 
Network. Accessed September 1, 2016.

http://jama.jamanetwork.com/article.aspx?articleid=1677374.

148. Domann E., G. Hong, C. Imirzalioglu, et al. 2003. Culture-Independent Identification of Pathogenic Bacteria and Polymicrobial Infections in the Genitourinary Tract of Renal Transplant Recipients. J. Clin. Microbiol. 41: 5500-5510.

149. Andersson P., M. Klein, R.A. Lilliebridge, et al. 2013. Sequences of multiple bacterial genomes and a Chlamydia trachomatis genotype from direct sequencing of DNA derived from a vaginal swab diagnostic specimen. Clin. Microbiol. Infect. 19: E405-E408.

150. Street T.L., N.D. Sanderson, B.L. Atkins, et al. 2017. Molecular diagnosis of orthopaedic device infection direct from sonication fluid by metagenomic sequencing. J. Clin. Microbiol. JCM.00462-17.

151. Zeller V., A. Ghorbani, C. Strady, et al. 2007. Propionibacterium acnes: An agent of prosthetic joint infection and colonization. J. Infect. 55: 119-124.

152. Osmon D.R., E.F. Berbari, A.R. Berendt, et al. 2013. Diagnosis and Management of Prosthetic Joint Infection: Clinical Practice Guidelines by the Infectious Diseases Society of America. Clin. Infect. Dis. 56: e1-e25.

153. Leggett R.M., C. Alcon-Giner, D. Heavens, et al. 2017. Rapid MinION metagenomic profiling of the preterm infant gut microbiota to aid in pathogen diagnostics. bioRxiv 180406.

154. Votintseva A.A., P. Bradley, L. Pankhurst, et al. 2017. Same-Day Diagnostic and Surveillance Data for Tuberculosis via Whole-Genome Sequencing of Direct Respiratory Samples. J. Clin. Microbiol. 55: 1285-1298.

155. Kerremans J.J., P. Verboom, T. Stijnen, et al. 2008. Rapid identification and antimicrobial susceptibility testing reduce antibiotic use and accelerate pathogen-directed antibiotic use. $J$. Antimicrob. Chemother. 61: 428-435.

156. Kollef M.H. 2000. Inadequate Antimicrobial Treatment: An Important Determinant of Outcome for Hospitalized Patients. Clin. Infect. Dis. 31: S131-S138.

157. Hyle E.P., A.D. Lipworth, T.E. Zaoutis, et al. 2005. Impact of Inadequate Initial Antimicrobial Therapy on Mortality in Infections Due to Extended-Spectrum $\beta$-Lactamase-Producing Enterobacteriaceae: Variability by Site of Infection. Arch. Intern. Med. 165: 1375-1380.

158. Mortality and delay in effective therapy associated with extended-spectrum $\beta$-lactamase production in Enterobacteriaceae bacteraemia: a systematic review and meta-analysis | Journal of Antimicrobial Chemotherapy | Oxford Academic. Accessed November 21, 2017. https://academic.oup.com/jac/article/60/5/913/2357962.

159. Davis J.J., S. Boisvert, T. Brettin, et al. 2016. Antimicrobial Resistance Prediction in PATRIC and RAST. Sci. Rep. 6:27930.

This article is protected by copyright. All rights reserved. 
160. Bradley P., N.C. Gordon, T.M. Walker, et al. 2015. Rapid antibiotic-resistance predictions from genome sequence data for Staphylococcus aureus and Mycobacterium tuberculosis. Nat.

Commun. 6: 10063.

161. Chen L., J. Yang, J. Yu, et al. 2005. VFDB: a reference database for bacterial virulence factors. Nucleic Acids Res. 33: D325-D328.

162. Holt K.E., H. Wertheim, R.N. Zadoks, et al. 2015. Genomic analysis of diversity, population structure, virulence, and antimicrobial resistance in Klebsiella pneumoniae, an urgent threat to public health.Proc. Natl. Acad. Sci. 112: E3574-E3581.

163. Diago-Navarro E., L. Chen, V. Passet, et al. 2014. Carbapenem-Resistant Klebsiella pneumoniae Exhibit Variability in Capsular Polysaccharide and Capsule Associated Virulence Traits J. Infect. Dis. 210: 803-813.

164. Yang JY., S. Brooks, J.A. Meyer, et al. 2013. Pan-PCR, a Computational Method for Designing Bacterium-Typing Assays Based on Whole-Genome Sequence Data. J. Clin. Microbiol. 51: $752-758$.

165. Bowers J.R., D. Lemmer, J.W. Sahl, et al. 2016. KlebSeq: A Diagnostic Tool for Surveillance, Detection, and Monitoring of Klebsiella pneumoniae. J. Clin. Microbiol. JCM.00927-16.

166. Fricke W.F. \& D.A. Rasko. 2014. Bacterial genome sequencing in the clinic: bioinformatic challenges and solutions. Nat. Rev. Genet. 15: 49-55.

167. Zou Q.-H., R.-Q. Li, G.-R. Liu, et al. 2016. Genotyping of Salmonella with lineage-specific genes: correlation with serotyping. Int. J. Infect. Dis. 49: 134-140.

168. Wilson M.R., S.N. Naccache, E. Samayoa, et al. 2014. Actionable Diagnosis of Neuroleptospirosis by Next-Generation Sequencing. N. Engl. J. Med. 370: 2408-2417.

169. Bonten M.J.M., B.J. Kullberg, R. van Dalen, et al. 2000. Selective digestive decontamination in patients in intensive care. J. Antimicrob. Chemother. 46: 351-362.

170. Shelburne S.A., J. Kim, J.M. Munita, et al. 2017. Whole-Genome Sequencing Accurately Identifies Resistance to Extended-Spectrum $\beta$-Lactams for Major Gram-Negative Bacterial Pathogens. Clin. Infect. Dis. 65: 738-745.

171. Quick J., P. Ashton, S. Calus, et al. 2015. Rapid draft sequencing and real-time nanopore sequencing in a hospital outbreak of Salmonella. Genome Biol. 16: 114.

172. Quick J,, N.J.Loman, S. Duraffour, et al. 2016. Real-time, portable genome sequencing for Ebola surveillance. Nature 530: nature16996.

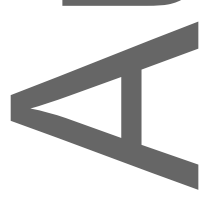

This article is protected by copyright. All rights reserved. 
Table 1. Open questions in the genomic epidemiology of resistant Gram-negatives

\begin{tabular}{|c|c|}
\hline \multirow{4}{*}{$\begin{array}{l}\text { Hospital epidemiology and } \\
\text { outbreak investigation }\end{array}$} & $\begin{array}{l}\text { What determines the structure of transmission networks for different } \\
\text { pathogens in different types of healthcare settings? }\end{array}$ \\
\hline & $\begin{array}{l}\text { What are the patient characteristics and clinical practices that affect } \\
\text { nosocomial transmission? }\end{array}$ \\
\hline & $\begin{array}{l}\text { What should be the standard best practices and analytical frameworks } \\
\text { for different types of genomic epidemiology investigations? }\end{array}$ \\
\hline & $\begin{array}{l}\text { What laboratory capacity for genomics should clinical microbiology, } \\
\text { public health, or research labs have for real-time epidemiological } \\
\text { investigations? }\end{array}$ \\
\hline \multirow{3}{*}{ Regional epidemiology } & $\begin{array}{l}\text { What are the clinical and epidemiological factors that affect regional } \\
\text { MDRO prevalence? }\end{array}$ \\
\hline & $\begin{array}{l}\text { What are the networks on which different MDROs spread between } \\
\text { regional healthcare facilities? }\end{array}$ \\
\hline & $\begin{array}{l}\text { How can genomics be integrated into public health workflows to affect } \\
\text { real-time outbreak control and guide regional interventions? }\end{array}$ \\
\hline \multirow{3}{*}{$\begin{array}{l}\text { Evolution and dissemination } \\
\text { of clonal lineages }\end{array}$} & $\begin{array}{l}\text { What genomic and epidemiological factors lead to the success of } \\
\text { epidemic lineages? }\end{array}$ \\
\hline & $\begin{array}{l}\text { How does sampling bias affect our understanding of the genetic and } \\
\text { epidemiological factors underlying the emergence of epidemic } \\
\text { lineages? }\end{array}$ \\
\hline & $\begin{array}{l}\text { Can we predict the epidemic potential of emergent lineages and } \\
\text { intervene to prevent their spread? }\end{array}$ \\
\hline \multirow{5}{*}{$\begin{array}{l}\text { Evolution of antibiotic } \\
\text { resistance }\end{array}$} & $\begin{array}{l}\text { Can we predict the epidemiological significance of resistance that } \\
\text { emerges within patients? }\end{array}$ \\
\hline & $\begin{array}{l}\text { How is resistance emergence influenced by treatment strategy and } \\
\text { patient characteristics? }\end{array}$ \\
\hline & $\begin{array}{l}\text { What influences the rate of horizontal transfer of resistance to MDROs } \\
\text { in patients? }\end{array}$ \\
\hline & $\begin{array}{l}\text { Which potential reservoirs of antibiotic resistance are sources and } \\
\text { which are sinks? }\end{array}$ \\
\hline & What is the direction of transferrable resistance flow between different \\
\hline
\end{tabular}

This article is protected by copyright. All rights reserved. 


\begin{tabular}{|l|l|}
\hline & reservoirs? \\
\hline \multirow{2}{*}{ Clinical diagnostics } & $\begin{array}{l}\text { Can patient outcomes be improved by implementing real-time genomic } \\
\text { diagnostics in clinical microbiology laboratories? }\end{array}$ \\
\cline { 2 - 2 } & $\begin{array}{l}\text { What is the capacity for genomics to reduce turnaround time for } \\
\text { antibiotic susceptibility testing and initiation of appropriate therapy? }\end{array}$ \\
\cline { 2 - 2 } & $\begin{array}{l}\text { How can genomics most effectively supplement phenotypic assays } \\
\text { given limitations in prediction of novel resistance alleles? }\end{array}$ \\
& $\begin{array}{l}\text { What is the value added of real-time genomic epidemiology } \\
\text { investigations versus designed retrospective studies of transmission? }\end{array}$ \\
\hline
\end{tabular}
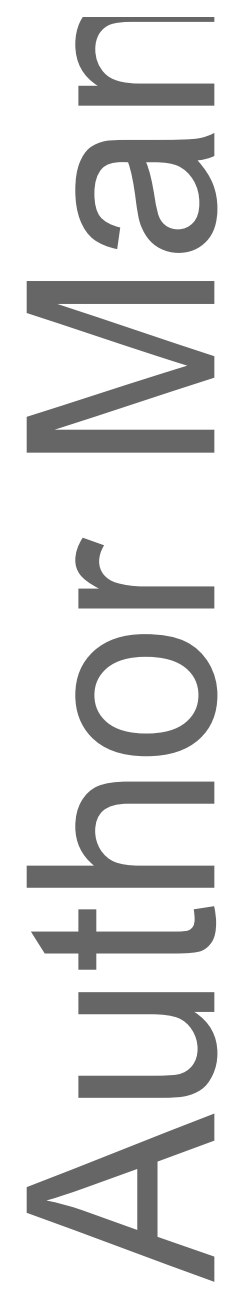

This article is protected by copyright. All rights reserved. 